\title{
Iatrogenic rupture of undiagnosed blood blister-like aneurysm during aneurysmal neck clipping
}

\author{
Sungmin Joo ${ }^{1}$, Mae-Hwa Kang ${ }^{1}$, Taewan Lim² ${ }^{2}$, Soo Kyung Lee ${ }^{1}$, and Hyung-Sik Hwang ${ }^{3}$ \\ ${ }^{1}$ Department of Anesthesiology and Pain Medicine, Hallym University Sacred Heart Hospital, Anyang, Departments of \\ ${ }^{2}$ Anesthesiology and Pain Medicine, ${ }^{3}$ Neurosurgery, Hallym University Dongtan Sacred Heart Hospital, Hwaseong, Korea
}

A 64-year-old, $49 \mathrm{~kg}, 155 \mathrm{~cm}$, female was admitted to the hospital with nausea and headache. She had hypertension on medications for 16 years. She maintained blood pressure (BP) of $170 / 80 \mathrm{mmHg}$ and heart rate (HR) of 60 beats/min on admission. She was alert and oriented but acute subarachnoid hemorrhage was suspected. Glasgow coma scale score was 4/5/6. Laboratory examinations, chest radiograph, and electrocardiogram did not show specific abnormalities. A preoperative 3-dimensional computed tomography (CT) angiogram showed a $3 \mathrm{~mm}$ sized saccular aneurysm of right posterior communicating artery (PComA), assessed by a neuroradiologist (Fig. 1A). She was scheduled for emergency neck clipping under general anesthesia. On arrival at the operating room, BP and HR were 140/80
$\mathrm{mmHg}$ and 98 beats/min. Induction of anesthesia was achieved with propofol and remifentanil using a target-controlled infusion machine, and rocuronium. After intubation, a 20-gauge catheter in the left radial artery and a triple-lumen catheter in the left subclavian vein were placed. Maintenance of anesthesia was achieved with propofol and remifentanil. Vital signs were stable with BP of 120/60 mmHg, HR of 82 beats/min, and central venous pressure (CVP) of $13 \mathrm{mmHg} 40$ minutes after induction. Pterional approach was performed at the supine position. The dura was tense and pale. Mild brain bulging was confirmed. At the dorsal wall of right internal carotid artery (ICA) bifurcation, the aneurysm was identified as a blood blister-like aneurysm (BBA) by the neurosurgeon, unlike preoperative di-
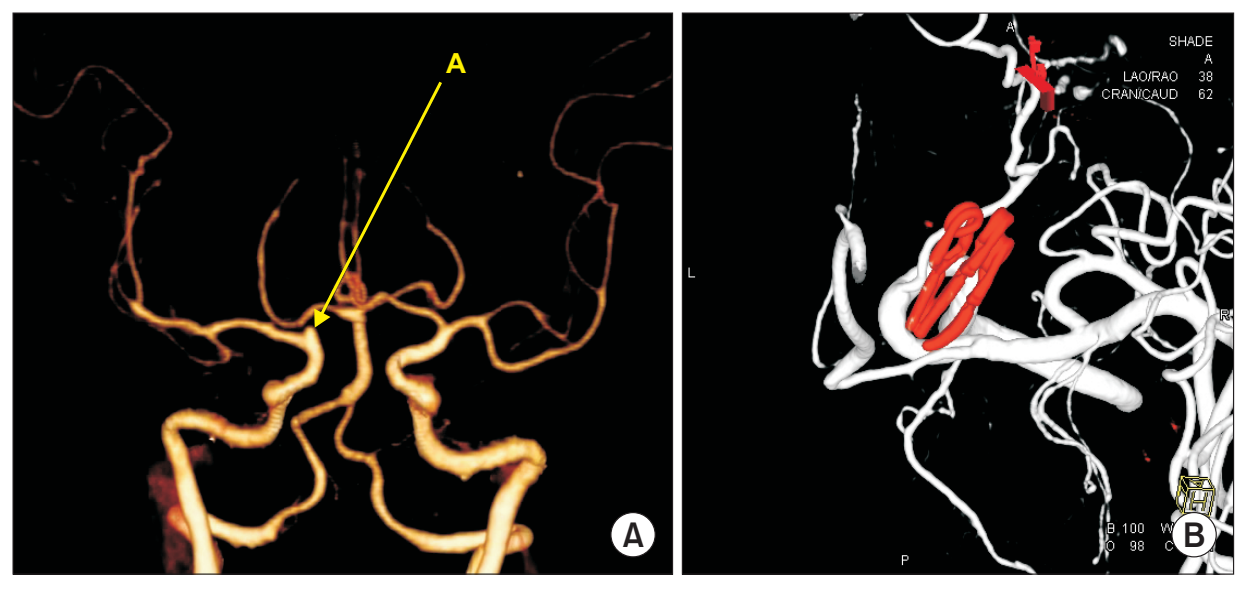

Fig. 1. (A) Preoperative 3-dimensional computed tomography angiogram showing small aneurysm at the right internal carotid artery bifurcation. A: Blood blister-like aneurysm. (B) Postoperative transfemoral cerebral angiography 3-dimensional image showing clipping.

Corresponding author: Taewan Lim, M.D., Department of Anesthesiology and Pain Medicine, Hallym University Dongtan Sacred Heart Hospital, 7, Keunjaebong-gil, Hwaseong 445-170, Korea. Tel: 82-31-8086-2283, Fax: 82-31-8086-2029, E-mail: ltwhanzo@naver.com

(c) This is an open-access article distributed under the terms of the Creative Commons Attribution Non-Commercial License (http:// creativecommons.org/licenses/by-nc/3.0/), which permits unrestricted non-commercial use, distribution, and reproduction in any medium, provided the original work is properly cited. 
agnosis. The aneurysm had broad base with a $3 \mathrm{~mm}$-sized dome and a $2 \mathrm{~mm}$-sized neck, and the aneurysmal sac with severe atherosclerotic change. When the neurosurgeon investigated aneurysmal sac with a dissector, the aneurysm was ruptured accidentally. Shortly after rupture, BP dropped to $70 / 40 \mathrm{mmHg}$ and HR increased 115 beats/min. CVP decreased to $8 \mathrm{mmHg}$. Temporary clipping was delayed for 15 minutes due to unskilled scrub nurse, surgical field flooding, and thin walled-BBA with broad base. Maintenance of BP was failed for 15 minutes regardless of massive colloid solution, vasopressin, and norepinephrine infusion. It was impossible to clip aneurysm neck due to surgical field flooding and broad-based neck. Eventually, other experienced neurosurgeon participated in the surgery. Temporary clipping was performed at the distal and proximal ICA and Sundt clip graft (Codman \& Shurtleff Inc., Raynham, MA, USA) was applied. However, bleeding did not stop completely. Yasargil $^{\circledR}$ straight clips (Aesculap Inc., San Francisco, CA, USA) were applied to the dissecting site over 20 minutes and bleeding finally stopped (Fig. 1B). Estimated blood loss was 2,500 ml and urine output was $2,000 \mathrm{ml}$. 5 pints of packed RBCs, 5 units of fresh frozen plasma, 2,000 ml of colloid solution, and 2,900 $\mathrm{ml}$ of crystalloid solution were administered. When surgery was ended, vital signs became stable. She was transferred to the intensive care unit under intubated state. On the first postoperative day, there was intracranial hemorrhage in left basal ganglia on CT. On the eighth postoperative day, there was right frontal lobe infarction on CT, and right hemiplegia due to intracranial hemorrhage in left basal ganglia was diagnosed. Triple- $\mathrm{H}$ therapy was performed after surgery and cerebral vasospasm did not occur.

BBAs are hazardous and account for $0.3-1 \%$ of intracranial aneurysms [1]. BBAs show small size, thin wall, broad-base, and mostly non-branching sites of the supraclinoid ICA [2,3]. Atherosclerosis and hemodynamic stress may be essential for BBAs formation [4]. However, the pathogenesis might be still unclear [3]. Diagnosis of BBAs is not feasible than saccular aneurysm despite imaging. Less impressive angiographic findings on magnetic resonance angiography (MRA) and CT angiography of BBAs might result in misdiagnosis [3]. Sometimes, angiographic configurations change from blister-like form to saccular form on serial images and grow rapidly over a short period [5]. Because definitive diagnosis of BBAs should depend on histopathological study [3], it is difficult for practitioners to diagnose BBAs before craniotomy. Conventional angiography should be considered in patients with subarachnoid hemorrhage and negative MRA or CT angiography. All four vessel territories should be checked with 3-dimensional techniques [3]. In our case, preoperative 3-dimensional CT-angiography was performed by the neuroradiologist. The aneurysm was diagnosed as a saccular aneurysm of right PComA, unlike the neurosurgeon's diagnosis on the surgical field (a BBA of right ICA bifurcation). Even when intracranial aneurysm is found in the site other than the ICA, we should consider misdiagnosis because undiagnosed and unexpected BBAs cause more critical circumstances than saccular aneurysm. Various therapeutic strategies have been introduced for BBAs. Endovascular stenting techniques is now the first choice for BBAs [3] and neurosurgical clipping should be reserved for cases which endovascular treatment cannot be applicable due to technical or neuroradiological experiences. Plans against BBAs rupture and cerebral protection against cerebral ischemia should be prepared. Trapping, wrapping, and revascularization might be mandatory so as not to compromise the cerebral blood flow. Although temporary clipping is essential for ruptured aneurysm, clipping of BBAs is not feasible due to fragile wall and may rupture on the first manipulation unlike saccular aneurysm [3]. In our case, repetitive temporary clipping was tried but BBA with thin and fragile wall, small size, and wide neck made temporary clipping difficult and led to laceration, massive bleeding, and hemodynamic instabilities. We never suspected BBAs until the neurosurgeon found morphological characteristics of BBAs. Meticulous radiological interpretation is important but morphological ambiguities of BBAs make management difficult $[3,5]$. Particularly, while neurosurgeons should focus on the surgical field, anesthesiologists might be overconfident in preoperative diagnosis and perform anesthetic management for saccular aneurysms, without considering BBAs. Neck clipping of BBAs is more difficult and dangerous than saccular aneurysms due to morphological characteristics and might result in anesthetic mishaps despite hemodynamic support. Anesthesiologists should prepare against conversion of diagnosis from saccular aneurysms to BBAs and massive bleeding due to iatrogenic rupture. In addition, it is more difficult to clip ruptured BBAs than saccular aneurysms and more time is required for clipping, bleeding control, and hemodynamic support of BBAs. Anesthesiologists should exchange opinions with neurosurgeons about findings on the surgical field.

\section{References}

1. McLaughlin N, Laroche M, Bojanowski MW. Surgical management of blood blister-like aneurysms of the internal carotid artery. World Neurosurg 2010; 74: 483-93. 
2. Doorenbosch X, Harding M. Primary treatment of a blood-blister-like aneurysm of the internal carotid artery with Guglielmi detachable coil embolisation. J Clin Neurosci 2008; 15: 1276-9.

3. Regelsberger J, Matschke J, Grzyska U, Ries T, Fiehler J, Köppen J, et al. Blister-like aneurysms--a diagnostic and therapeutic challenge. Neurosurg Rev 2011; 34: 409-16.

4. Tekkök IH, Bakar B. Ruptured blister-like aneurysm of distal internal carotid artery: a distinct entity. Turk Neurosurg 2008; $18: 439-45$.

5. Kim JH, Kwon TH, Kim JH, Park YK, Chung HS. Internal carotid artery dorsal wall aneurysm with configurational change: Are they all false aneurysms? Surg Neurol 2006; 66: 441-3. 